\title{
Abordagem estatística na validação retrospectiva do processo de fabricação de mistura polivitamínica
}

\author{
Aline Lobato Anholeto Vissotto, Gislei Lunkes Vilela, Elvira Blanco Casais, Hélide Stefani, \\ Valeska Martinello, Nelson dos Santos Junior, Maurício Sampaio, Nádia Araci Bou-Chacra*
}

Departamento de Farmácia, Faculdade de Ciências Farmacêuticas, Universidade de São Paulo

*Correspondência:

N. Bou-Chacra

Departamento de Farmácia

Faculdade de Ciências Farmacêuticas

Universidade de São Paulo

R. Prof. Lineu Prestes, 580

05314-970 - São Paulo - SP, Brasil

E-mail: chacra@usp.br
As exigências legais relativas à rotulagem de alimentos, em especial aqueles constituídos de polivitamínicos e minerais destinados a lactentes e crianças de primeira infância, requerem processos de fabricação que assegurem os valores declarados. Dessa forma, os fornecedores de mistura de polivitamínicos devem demonstrar que o processo de homogeneização dos pós conduz aos resultados esperados, conforme especificado. Os estudos de validação devem contemplar tal exigência e podem ser efetuados de forma prospectiva, concorrente ou retrospectiva. A abordagem retrospectiva, fundamentada na revisão e análise de registros históricos, pode ser efetuada no caso dos lotes considerados terem sido produzidos segundo os mesmos parâmetros operacionais e especificações. Assim sendo, o objetivo do presente trabalho foi validar o processo de fabricação de produto constituido pela mistura das seguintes vitaminas: cloridrato de piridoxina, nicotinamida, ácido ascórbico e pantotenato de cálcio, empregando abordagem retrospectiva. A análise estatística dos dados relativos ao teor de cada componente foi efetuada empregando gráficos de controle objetivando a avaliação da estabilidade do processo. Os índices de capacidade $C_{p}$ e $C_{p k}$ foram calculados após avaliação da distribuição dos dados empregando teste de Anderson-Darling. Todos os dados apresentaram distribuição normal exceto aqueles relativos ao teor de nicotinamida. Esses dados foram normalizados utilizando método proposto por Box-Cox (lambda igual a -4,24). Os índices de capacidade $\left(C_{p k}\right)$ obtidos foram 1,01, 1,14, 1,21 e 1,96, respectivamente, para cloridrato de piridoxina, nicotinamida, ácido ascórbico e pantotenato de cálcio. Tais resultados indicaram que o processo de mistura de polivitaminas pode ser considerado validado. Além disso, investigações adicionais visando à centralização da concentração teórica, na faixa relativa aos limites de controle, devem ser conduzidas.
Unitermos

- Polivitaminas

- Validação

- Estabilidade

- Normalidade

- Capacidade 


\section{INTRODUÇÃO}

A indústria de alimentos, de forma geral, obtém a mistura de polivitamínicos de fornecedores qualificados e adiciona tal mistura em fase específica de sua produção. No produto final deve ser comprovada a concentração declarada de cada componente, conforme exigência legal. Com referência à rotulagem nutricional de alimentos, a Resolução RDC n n $^{\circ} 60$ de 23 de dezembro de 2003 determinou a aderência das empresas às novas exigências a partir de 31 de julho de 2006. Tais exigências visam à harmonização de aspectos técnicos e legais objetivando o comércio de alimentos no âmbito do Mercosul, além de beneficiar o consumidor com as informações declaradas. A legislação inclui alimentos constituídos por polivitamínicos e minerais destinados a lactentes e crianças de primeira infância. Esses produtos podem ser usados para satisfazer necessidades particulares de alimentação determinadas por condições físicas ou fisiológicas e, ainda, por deficiências metabólicas.

Assim sendo, os fornecedores de mistura de polivitamínicos devem ser capazes de demonstrar que seus processos desempenham suas funções de forma consistente, permitindo que o produto final apresente as informações conforme declaradas. Dessa forma, são exigidos os estudos de validação do processo da mistura de vitaminas visando assegurar a produção de lotes uniformes, conforme as Boas Práticas de Fabricação (Brasil, 2003; United States, 1987).

Com relação às possíveis não-conformidades do processo de mistura de vitaminas, essas podem ser decorrentes de erros e/ou de excessiva variabilidade do processo. Considerando a possibilidade de erro, a adoção de procedimentos operacionais padrões adequados, periodicamente revisados, minimiza tal ocorrência. No que se refere à sua variabilidade, a capacidade do processo em produzir lotes que atendam às especificações requeridas deve ser determinada por meio de estudo sistematizado empregando ferramenta estatística.

Os estudos de validação constituem parte essencial das Boas Práticas de Fabricação e podem ser efetuados de forma concorrente, durante o processo de fabricação, de forma prospectiva, na fase de desenvolvimento do produto ou de forma retrospectiva, baseada na revisão e análise de registros históricos. No caso de validação retrospectiva, todos os lotes considerados devem ter sido produzidos segundo os mesmos parâmetros operacionais e especificações (Brasil, 2003). Quando possível a constatação de tal exigência, a abordagem retrospectiva apresenta como vantagem reduzido custo relativo à execução das análises, previamente concluídas na rotina de fabricação da mistura, além de elevado número de dados analíticos permitindo análise estatística consistente do processo de fabricação.
Assim sendo, o objetivo do presente trabalho foi validar o processo de fabricação de produto contendo mistura das seguintes vitaminas: cloridrato de piridoxina, nicotinamida, ácido ascórbico e pantotenato de cálcio empregando abordagem retrospectiva.

\section{MATERIAL E MÉTODOS}

\section{Material}

O material, objeto do presente estudo, foi constituído de mistura polivitamínica: cloridrato de piridoxina (vitamina B6, DSM Nutricional Products AG), nicotinamida (vitamina PP, DSM Nutricional Products Ltd), ácido ascórbico (vitamina C, DSM Nutricional Products Ltd, UK), pantotenato de cálcio (vitamina B5, DSM Nutricional Products Ltd, UK), ácido esteárico (Cropton Corporation, Witco Corporation e Dinalab Com. Imp. e Exportação Ltda), celulose microcristalina (Avicel ${ }^{\circledR}$ PH 102, Mase Prod. Quim. Farm. Ltda), estearato de magnésio (Meltec Com. \& Representações Ltda e Industria Química Anastácio SA) e dióxido de silício (Aerosil ${ }^{\circledR}$ 200, Degussa Brasil Ltda).

\section{Método}

\section{Produção dos lotes de mistura polivitamínica}

O fluxograma abaixo apresenta o processo de obtenção de lotes de $800 \mathrm{~kg}$ de mistura de polivitamínicas.

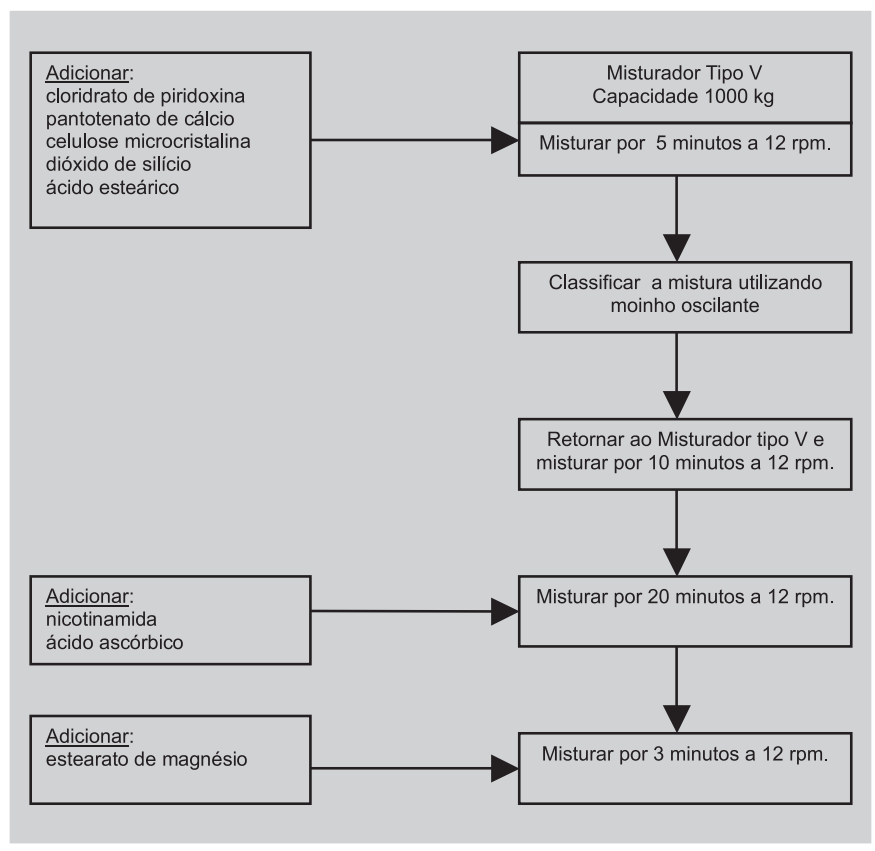

FIGURA 1 - Fluxograma representativo do processo de fabricação da mistura polivitamínica. 


\section{Determinação do teor de vitaminas}

$\mathrm{O}$ teor das vitaminas foi determinado empregando métodos validados descritos em monografia oficial (United States Pharmacopoeia, 2005). A amostragem foi efetuada no início, no meio e no fim do envase de caixa padrão contendo $25 \mathrm{~kg}$. A análise foi efetuada em duplicata.

\section{Análise estatística dos dados}

A análise estatística dos dados relativos aos 26 lotes produzidos foi efetuada empregando software Minitab ${ }^{\circledR}$ versão 14, conforme fluxograma apresentado na Figura 2.

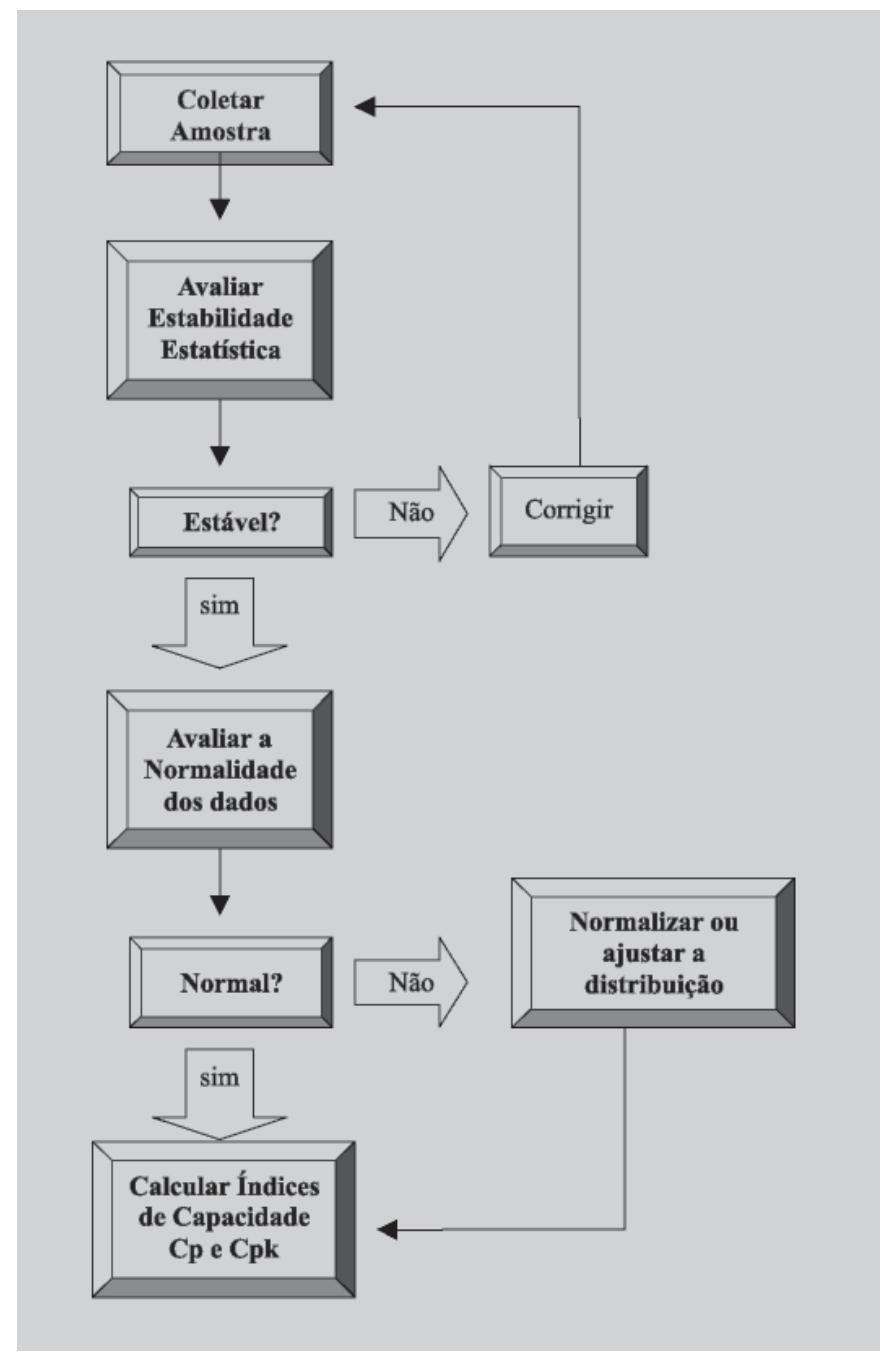

FIGURA 2 - Fluxograma referente às etapas relativas à determinação de estabilidade e capacidade do processo.

Avaliação da estabilidade do processo de mistura polivitamínica

Os limites de controle relativos aos gráficos das observações individuais $(\bar{x})$ foram calculados empregando as seguintes fórmulas:

$$
\begin{aligned}
& \text { LSC }=\overline{\bar{x}}+A_{2} \cdot \bar{R} \\
& \text { Linha_central }=\overline{\bar{x}} \\
& L I C=\overline{\bar{x}}-A_{2} \cdot \bar{R}
\end{aligned}
$$

Onde:

$\mathrm{A}_{2}=$ constante que se encontra tabulada em função do tamanho de amostra (Montgomery, 2004)

$\overline{\bar{x}}=$ média geral do processo, ou seja, dos lotes avaliados $\bar{R}=$ amplitude média dos lotes avaliados

Além do gráfico $\bar{x}$, a variabilidade do processo foi avaliada por meio dos valores das amplitudes amostrais $\mathrm{R}$ plotados em um gráfico de controle das amplitudes móveis (R), cuja fórmula dos limites de controle está apresentada abaixo:

$$
\begin{aligned}
& L S C=D_{4} \cdot \bar{R} \\
& \text { Linha_central }=\bar{R} \\
& L I C=D_{3} \cdot \bar{R}
\end{aligned}
$$

Onde:

$\mathrm{D}_{3}$ e $\mathrm{D}_{4}=$ constantes que se encontram tabuladas em função do tamanho de amostra (Montgomery, 2004).

\section{Avaliação da distribuição de probabilidade normal} dos dados

O teste de normalidade Anderson-Darling foi efetuado para verificar a probabilidade normal dos dados. O teste de aderência (Ramos, 1999) é baseado no cálculo da estatística $A$ definida como:

$$
A=A^{2} \times\left[1+\frac{0,75}{n}+\frac{2,25}{n^{2}}\right]
$$

Onde:

$$
A^{2}=-\sum_{I=1}^{n}\left[(2 i-1) \times \frac{\ln \Phi_{(x i)}+\ln \left(1-\Phi_{(x n+1-i)}\right)}{n}\right]-n
$$

$\phi=$ função de distribuição normal acumulada. Se $A$ exceder o valor crítico de 0,752 , ao nível de significância de $5 \%$, rejeita-se que a distribuição seja normal. $\mathrm{n}=$ tamanho da amostra. 
Para os dados primários que não apresentaram distribuição normal, quando submetidos ao teste de normalidade Anderson-Darling, foi efetuada a transformação dos mesmos empregando a abordagem gráfica e analítica do Box-Cox (Montgomery, 2004). A métrica de transformação selecionada é definida como:

$$
T(Y)=\left(Y^{\lambda}-1\right) / \lambda
$$

Onde:

$\mathrm{T}(\mathrm{Y})=$ dado transformado

$\mathrm{Y}=$ resposta da variável

$\lambda=$ parâmetro transformado, sendo para $\lambda=0, \mathrm{o}$ logaritmo dos dados.

\section{Determinação dos índices de capacidade do processo}

Os índices de capacidade a curto prazo $\left(C_{p}\right.$ e $\left.C_{p \mathrm{k}}\right)$ (Pearn, Lin, 2004) foram determinados para avaliação do processo de mistura de polivitamínica empregando as seguintes fórmulas:

$$
\begin{gathered}
C p=\frac{L S E-L I E}{6 \sigma_{c p}} \\
C p k=\text { mínimo }\left\{\frac{L S E-\mu}{3 \sigma_{c p}}, \frac{\mu-L I E}{3 \sigma_{c p}}\right\}
\end{gathered}
$$

Onde:

LSE $=$ limite superior de especificação

LIE $=$ limite inferior de especificação

$\mu=$ média do processo

$\sigma_{\mathrm{cp}}=$ desvio padrão de curto prazo.

\section{RESULTADOS E DISCUSSÃO}

A adição de dióxido de silício, do ácido esteárico assim como do estearato de magnésio teve como objetivo alcançar adequada fluidez da mistura de pós na etapa de envase do produto. No que se refere à adição de celulose microcristalina, esse componente foi utilizado como diluente. Com referência à mistura, o tempo estabelecido foi de 38 minutos para o cloridrato de piridoxina e para o pantotenato de cálcio e de 23 minutos para a nicotinamida e para o ácido ascórbico. A velocidade da mistura foi constante e igual a $12 \mathrm{rpm}$ (rotações por minuto), conforme fluxograma apresentado na Figura 1.

A Tabela I apresenta os dados relativos à determinação do teor de cada vitamina, nos 26 lotes avaliados. Na Figura 3a, os limites superior (LSC) e inferior (LIC) de con- trole foram estimados a partir das amplitudes móveis com nível de abrangência igual a 2 sendo, respectivamente, 11,495 e $9,416 \mathrm{~g} / \mathrm{kg}$. Esses valores se apresentaram dentro dos limites de especificação superior (LSE) e inferior (LIE) $(15,681$ e $9,410 \mathrm{~g} / \mathrm{kg})$. Tal resultado revelou que a variabilidade do processo permite atender as especificações adotadas para teor de cloridrato de piridoxina. Com referência à concentração, o valor teórico foi de $10,456 \mathrm{~g} / \mathrm{kg}$. Essa concentração foi similar à média obtida considerando os 26 lotes avaliados: 10,455 g/kg (Figura 3a). Além disso, não foram observadas causas especiais indicando, dessa forma, a estabilidade do processo, para essa vitamina.

A Figura $3 \mathrm{~b}$ apresenta o resultado do teste AndersonDarling para a avaliação da normalidade dos dados relativos ao teor de cloridrato de piridoxina. $\mathrm{O}$ valor $p$ igual a 0,098 (critério de aceitação $\geq 0,05$ ) mostrou que a distribuição dos dados para o teor dessa vitamina pode ser considerada normal.

Tendo em vista a estabilidade estatística do processo, assim como a distribuição normal dos dados para o cloridrato de piridoxina, as capacidades potencial e efetiva do processo foram estimadas ( $C p$ e $C p k$ ), conforme Figura $3 \mathrm{c}$. De forma geral, valores maiores que 1 indicam que o processo é capaz de atender à especificação adotada. No caso de valores inferiores a 1, pode-se inferir que o processo não apresenta consistência. Nesse caso, a qualidade dos sucessivos lotes produzidos pode variar conduzindo ao não atendimento às especificações. Embora valores maiores que 1 possam ser considerados adequados, no geral, têm sido propostos valores mínimos de 1,33 e, até 1,66 (DudekBurlikowska, 2005). Tais valores não possuem qualquer embasamento estatístico, mas constituem faixa de trabalho que busca assegurar menor probabilidade de produção de lotes com teor não previsto pelas especificações préestabelecidas.

Para o cloridrato de piridoxina, o valor para a capacidade efetiva do processo $\left(C_{p k}\right)$, com nível de significância de $95 \%$, foi igual a 1,01 . Porém, a capacidade potencial do processo $\left(C_{p}\right)$ foi de 3,02 . Tal diferença $(2,01)$ pode ser explicada em função da não-centralização da concentração teórica quando comparada aos limites de especificação adotados (Figura 3c). Foi possível observar a concentração deslocada para região próxima ao limite inferior (Figura 3a e Tabela II). Embora capaz, (variabilidade intrínseca do processo menor que os limites de especificação), a centralização da concentração teórica permitiria a produção de lotes com menor probabilidade de falha, ou seja, o processo apresentaria maior capacidade. No caso do cloridrato de piridoxina, 1274 lotes em 1 milhão podem apresentar teor abaixo do limite inferior de especificação considerando o índice de capacidade igual a 1,01 . No que se refere ao nú- 
TABELA I - Teor $(\mathrm{g} / \mathrm{kg})$ relativo às vitaminas: cloridrato de piridoxina, nicotinamida, ácido ascórbico e de pantotenato de cálcio nos 26 lotes avaliados

\begin{tabular}{|c|c|c|c|c|}
\hline \multirow[t]{2}{*}{ Lote } & \multicolumn{4}{|c|}{ Teor $(\mathrm{g} / \mathrm{kg})$} \\
\hline & cloridrato de piridoxina & nicotinamida & ácido ascórbico & pantotenato de cálcio \\
\hline 1 & 10,670 & 82,882 & 508,150 & 34,387 \\
\hline 2 & 10,620 & 82,679 & 515,389 & 35,339 \\
\hline 3 & 10,470 & 84,360 & 511,540 & 35,820 \\
\hline 4 & 10,390 & 79,386 & 489,225 & 34,999 \\
\hline 5 & 10,390 & 79,188 & 507,851 & 34,060 \\
\hline 6 & 10,260 & 80,016 & 514,529 & 34,423 \\
\hline 7 & 10,760 & 81,507 & 486,685 & 35,253 \\
\hline 8 & 9,760 & 80,606 & 519,118 & 36,792 \\
\hline 9 & 9,770 & 80,027 & 484,873 & 37,259 \\
\hline 10 & 10,360 & 81,462 & 535,220 & 35,108 \\
\hline 11 & 10,330 & 79,080 & 496,417 & 35,108 \\
\hline 12 & 11,430 & 84,290 & 499,006 & 35,108 \\
\hline 13 & 10,280 & 78,912 & 499,010 & 35,061 \\
\hline 14 & 10,610 & 79,576 & 526,917 & 35,061 \\
\hline 15 & 10,220 & 80,289 & 534,803 & 36,395 \\
\hline 16 & 10,540 & 84,688 & 537,474 & 37,625 \\
\hline 17 & 11,230 & 80,134 & 530,946 & 35,322 \\
\hline 18 & 10,660 & 84,711 & 531,966 & 35,912 \\
\hline 19 & 10,890 & 87,889 & 522,102 & 37,911 \\
\hline 20 & 10,550 & 82,483 & 494,340 & 35,311 \\
\hline 21 & 9,910 & 78,761 & 491,770 & 36,094 \\
\hline 22 & 9,620 & 86,104 & 500,133 & 34,879 \\
\hline 23 & 10,440 & 87,918 & 492,835 & 36,603 \\
\hline 24 & 10,440 & 87,918 & 492,835 & 36,603 \\
\hline 25 & 10,680 & 82,528 & 560,033 & 36,416 \\
\hline 26 & 10,560 & 85,737 & 462,100 & 34,674 \\
\hline LIE & 9,410 & 73,904 & 443,422 & 30,596 \\
\hline LSE & 15,684 & 123,173 & 739,037 & 50,994 \\
\hline $\mathrm{CT}$ & 10,456 & 82,115 & 492,691 & 33,996 \\
\hline
\end{tabular}

LIE: limite inferior de especificação; LSE: limite superior de especificação; CT: concentração teórica

mero de lotes com teor acima do limite de especificação, conforme resultado apresentado na Figura $3 \mathrm{c}$, nenhum lote em 1 milhão apresentaria tal resultado.

Os índices de longo prazo $\mathrm{P}_{p}$ e $\mathrm{P}_{p K}$, apesar de apresentados nas Figuras 3c, 4e, 5c e 6c, não foram utilizados na discussão dos resultados. Tais índices não apresentaram interpretação significativa na avaliação de capacidade de processo fundamentada na análise de registros históricos. Segundo Pearn e Lin (2004) e Stoumbos (2002), os índices $C_{p}$ e $C_{p k}$ são amplamente utilizados na determinação do desempenho de vários tipos de processos. Esses índices também foram utilizados em estudo proposto por Srikaeo et al. (2005) na avaliação de processo de fabricação de biscoitos a base de trigo.
Com referência ao teor de nicotinamida, os limites superior (LSC) e inferior (LIC) de controles calculados (Figura 4a) foram de, respectivamente, $89,90 \mathrm{~g} / \mathrm{kg}$ e 74,96 g/ $\mathrm{kg}$. Esses valores se apresentaram dentro dos limites de especificação superior (LSE igual a 123,17 g/kg) e inferior (LIE igual a 73,90 g/kg). A análise do gráfico de controle das observações individuais revelou ausência de causas especiais. Esse resultado indicou que a variabilidade do processo pode ser considerada aceitável, para teor de nicotinamida. Portanto, o processo pode ser considerado estável. No que se refere à concentração teórica, foi requerido valor igual a $82,12 \mathrm{~g} / \mathrm{kg}$. Esse valor foi próximo à média obtida considerando os 26 lotes avaliados: $82,43 \mathrm{~g} / \mathrm{kg}$ (Figura 4a). 

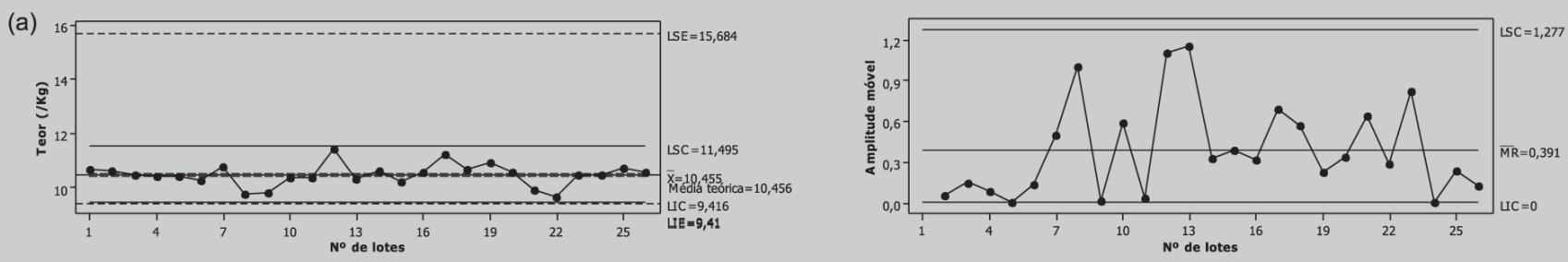

(b)

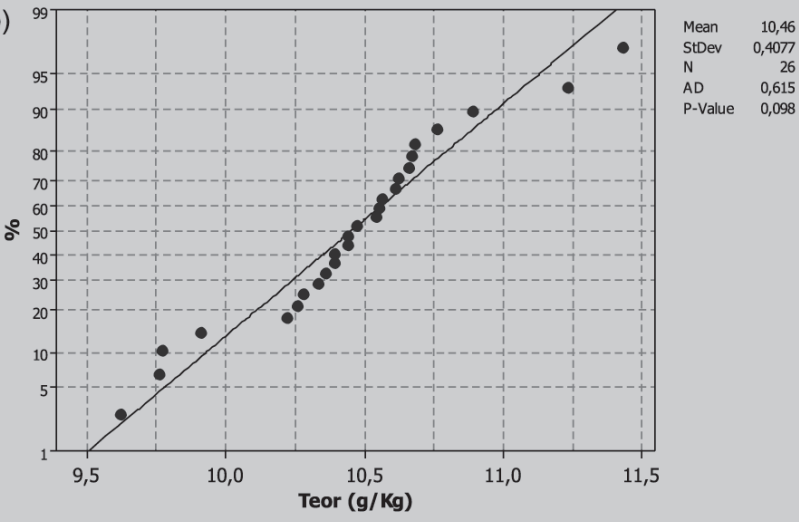

(c)

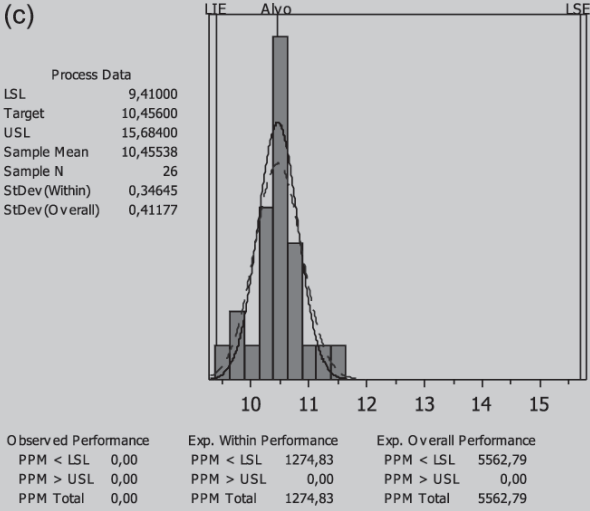

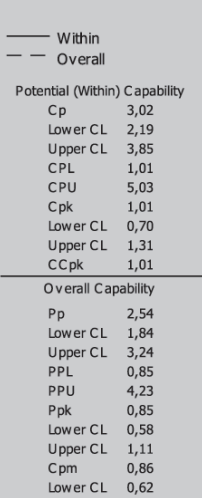

FIGURA 3 - Gráficos de controle das observações individuais e das amplitudes móveis (a), gráfico da probabilidade normal (b) e gráfico da capacidade do processo (c) relativo ao teor de cloridrato de piridoxina na mistura polivitamínica.

A Figura $4 \mathrm{~b}$ apresenta o resultado do teste AndersonDarling para a avaliação da normalidade dos dados relativos ao teor de nicotinamida. O valor $p$ igual a 0,044 (critério de aceitação e" 0,05 ) revelou a necessidade de transformação dos dados. Conforme Montgomery (2004), a interpretação dos índices de capacidade de determinado processo é fundamentada em distribuição normal da saída do processo. O valor de $p$ para os dados transformados, segundo Box-Cox, empregando $\lambda$ igual a - 4,24 estão apresentados nas Figuras $4 \mathrm{c}$ e $4 \mathrm{~d}$. Após a transformação desses dados, o valor obtido de $p$ foi de 0,098 (critério de aceitação $\geq 0,05$ ).

Os dados transformados foram utilizados para o cálculo da capacidade efetiva do processo $\left(C_{p k}\right)$ para o teor de nicotinamida, com nível de significância de $95 \%$. O resultado foi igual a 1,14 , enquanto que a capacidade potencial do processo $\left(C_{p}\right)$ foi cerca de três vezes maior $(3,30)$. Conforme mencionado anteriormente, a diferença significativa entre os valores de $C_{p}$ e $C_{p k}(2,16)$ refere-se à nãocentralização da concentração teórica quando comparada ao limites de especificação adotados (Figura 4e). Apesar da diferença entre os índices de capacidade, o processo pode ser considerado capaz para o teor de nicotinamida. No caso dessa vitamina, foi observada redução na probabilidade de ocorrência de resultados abaixo do LIE em comparação ao teor de cloridrato de piridoxina: 309 lotes em 1 milhão de oportunidades. Tal resultado pode ser atribuído ao maior índice $C_{p k}(1,14)$ para o teor de nicotinamida se comparado ao valor de 1,01 obtido para teor de cloridrato de piridoxina.

Com referência aos teores de ácido ascórbico e pantotenato de cálcio, os limites superior (LSC) e inferior (LIC) de controles calculados (Figuras 5a e 6a) apresentaram valores de, respectivamente, $563,9 \mathrm{~g} / \mathrm{kg}$ e $455,0 \mathrm{~g} / \mathrm{kg}$ para ácido ascórbico e 38,25 g/ $/ \mathrm{kg}$ e $33,09 \mathrm{~g} / \mathrm{kg}$ para o pantotenato de cálcio. Tais valores se apresentaram dentro dos limites de especificação superior e inferior, segundo descrito na Tabela I. Na análise do gráfico de controle das observações individuais não foram detectadas causas especiais para ambas às características: teores relativos ao ácido ascórbico e ao pantotenato de cálcio (Figuras 5a e 6a). Esse comportamento indicou a estabilidade do processo para essas vitaminas. As médias obtidas se apresentaram próximas à concentração teórica: $509,4 \mathrm{~g} / \mathrm{kg}$ para o ácido ascórbico e 35,67 g/kg para pantotenato de cálcio (Figuras $5 a$ e $6 a)$.

Nas Figuras 5b e 6 b estão apresentados os resultados do teste Anderson-Darling para a avaliação da normalidade dos dados relativos, respectivamente, ao teor de ácido ascórbico e ao teor de pantotenato de cálcio. Os valores de $p$ obtidos foram 0,467 para o ácido ascórbico e 0,077 para o pantotenato de cálcio (critério de aceitação $\geq 0,05$ ). Tais 
resultados revelaram distribuição normal dos dados para ambos os teores.

No que se refere ao ácido ascórbico, o valor obtido para a capacidade efetiva do processo $\left(C_{p k}\right)$, com nível de significância de $95 \%$, foi igual a 1,21 . No entanto, a capacidade potencial do processo $\left(C_{p}\right)$ foi de 2,71 , conforme Figura $5 \mathrm{c}$ e Tabela II. Esse resultado revelou que a concentração teórica se apresenta deslocada para região próxima ao LIE. No entanto, o processo pode ser considerado capaz de atender às especificações adotadas $\left(C_{p k}>1\right)$. A Figura $5 \mathrm{c}$ revelou que a probabilidade de ocorrência de resultados abaixo do LIE para teor de ácido ascórbico é de 138 lotes em 1 milhão de oportunidades e nenhum lote em 1 milhão apresentaria resultado superior ao LSE.

$O$ valor obtido para a capacidade efetiva do teor de pantotenato de cálcio $\left(C_{p k}\right)$, com nível de significância de (a)
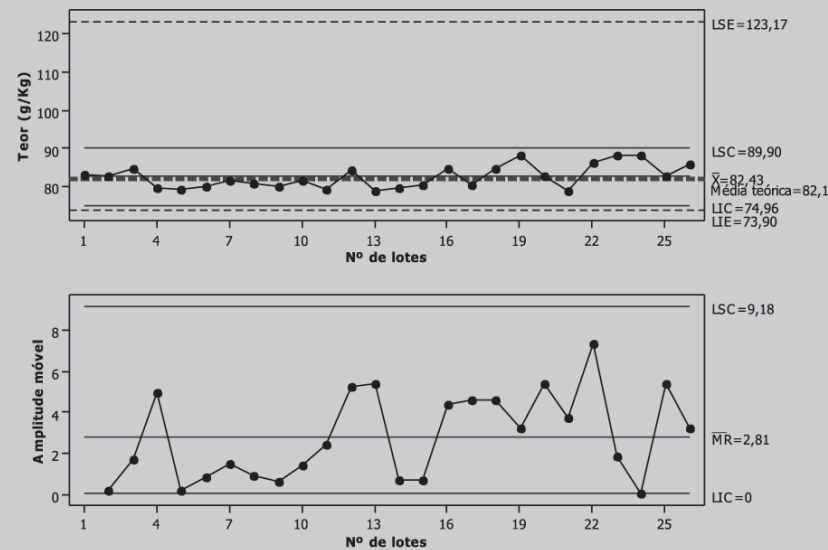

(b)

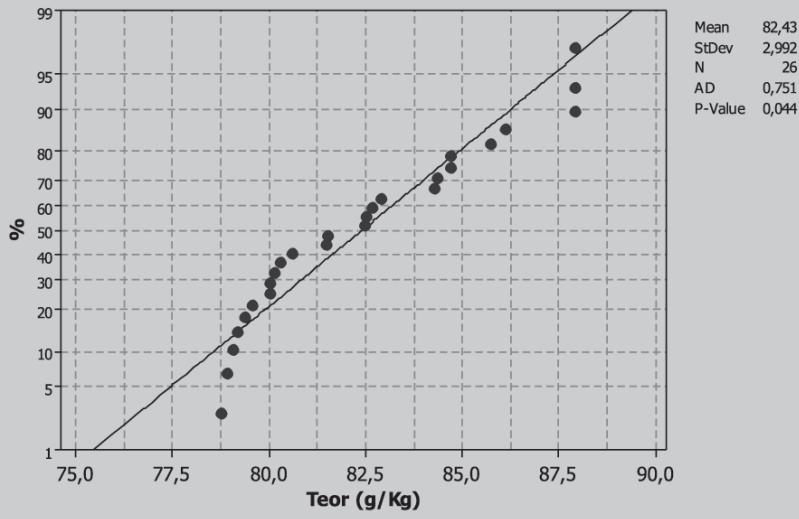

(c)

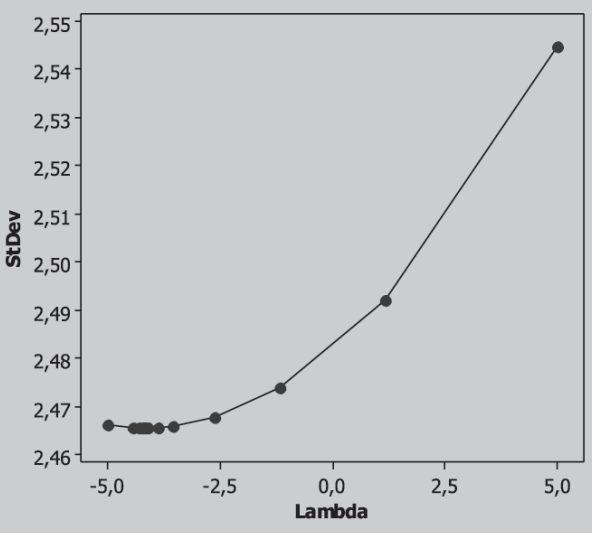

(d)

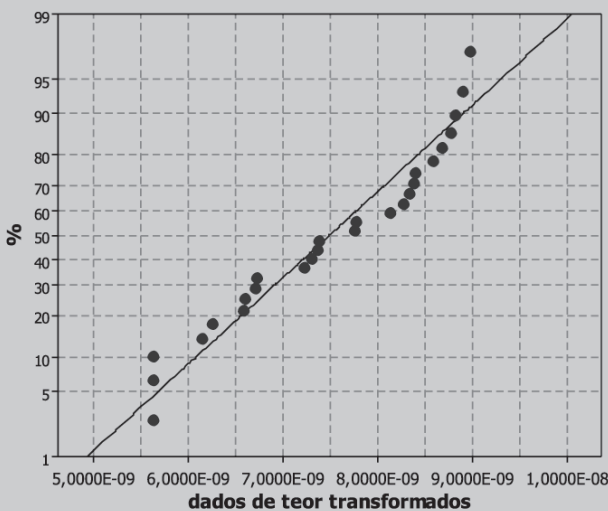

Lambda (using 95,0\% confidence) Estimate $\quad-4,24362$ Lower $\mathrm{CL}$ Best Value $\quad-4,24362$ Mean $\quad 7,495574 E-09$ StDev $\quad 1,101898 E-09$

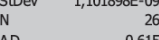

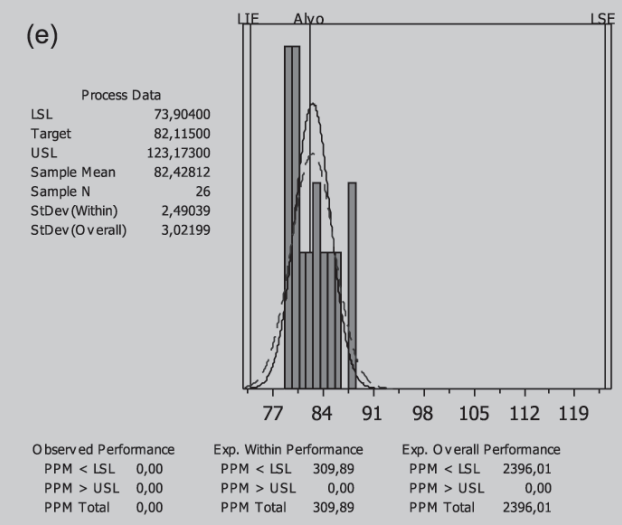

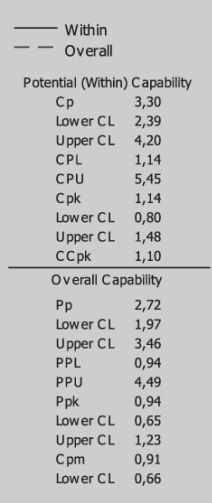

FIGURA 4 - Gráficos de controle das observações individuais e das amplitudes móveis (a), gráfico da probabilidade normal (b), curva do desvio padrão versus lambda (c), gráfico da probabilidade normal para $\lambda=-4,24$ (d) e gráfico da capacidade do processo (e) relativo ao teor de nicotinamida na mistura polivitamínica. 
(a)
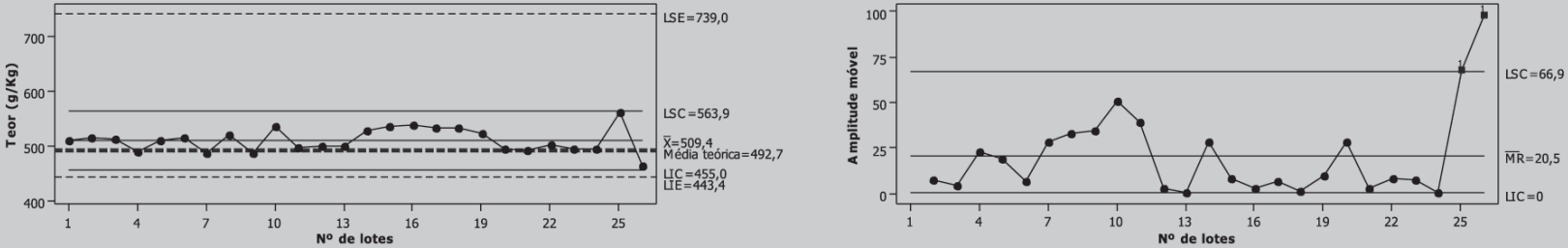

1 = resultado acima dos 3 desvios

(b)

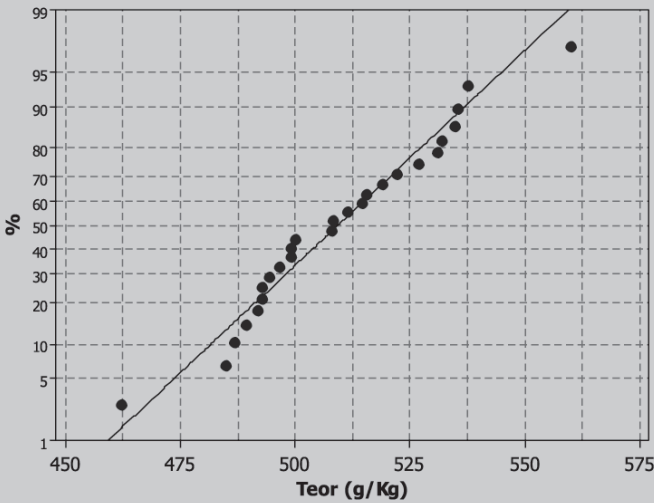

$\begin{array}{lr}\text { Mean } & 509,4 \\ \text { StDev } & 21,53 \\ \text { N } & 26 \\ \text { AD } & 0,341 \\ \text { P-Value } & 0,467\end{array}$

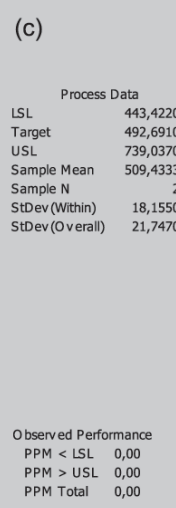

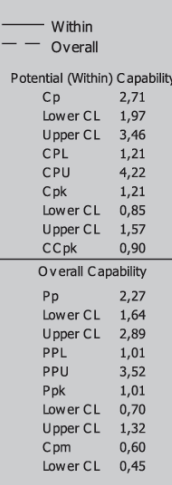

FIGURA 5 - Gráficos de controle das observações individuais e das amplitudes móveis (a), gráfico da probabilidade normal (b) e gráfico da capacidade do processo (c) relativo ao teor de ácido ascórbico na mistura polivitamínica.

(a)

(b)

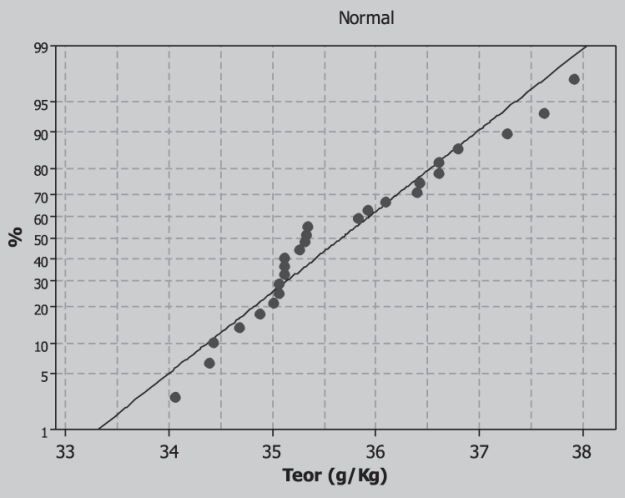
$\begin{array}{lr}\text { Mean } & 35,67 \\ \text { StDev } & 1,016 \\ \text { N } & 26 \\ \text { AD } & 0,656 \\ \text { P-Value } & 0,077\end{array}$
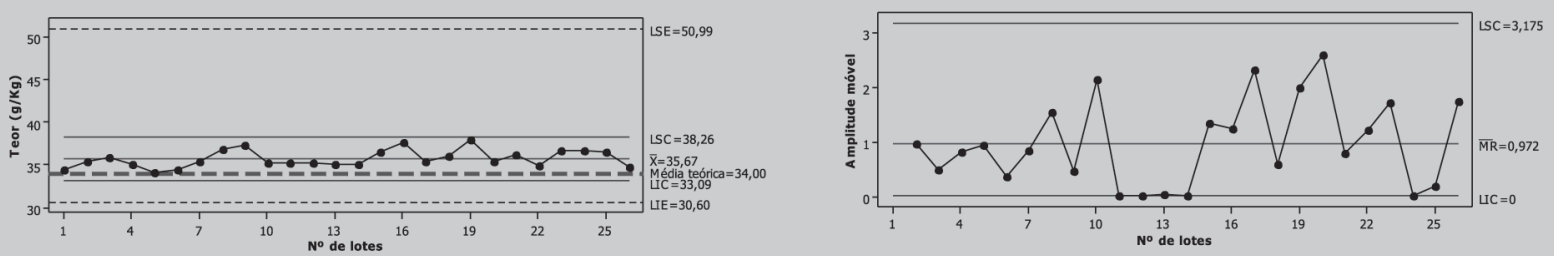

(c)

FIGURA 6 - Gráficos de controle das observações individuais e das amplitudes móveis (a), gráfico da probabilidade normal (b) e gráfico da capacidade do processo (c) relativo ao teor ao teor de pantotenato de cálcio na mistura polivitamínica. 
TABELA II - Índices de capacidades (Cp, CPL, CPU e Cpk) e probabilidade de falha no processo de mistura polivitamínica: cloridrato de piridoxina, nicotinamida, ácido ascórbico e pantotenato de cálcio

\begin{tabular}{lcccc}
\hline & cloridrato de piridoxina & nicotinamida & ácido ascórbico & pantotenato de cálcio \\
\hline Cp & 3,02 & 3,30 & 2,71 & 3,95 \\
CPL & 1,01 & 1,14 & 1,21 & 1,96 \\
CPU & 5,03 & 5,45 & 4,22 & 5,03 \\
Cpk & 1,01 & 1,14 & 1,21 & 1,96 \\
PPM total & 1274,83 & 309,89 & 138,46 & 0,37 \\
\hline
\end{tabular}

PPM: partes por milhão

$95 \%$, foi igual a 1,96 e, capacidade potencial $\left(C_{p}\right)$ de 3,95 (Figura 6c e Tabela II). Nesse caso, o teor do pantotenato de cálcio apresentou maior centralização em relação à concentração teórica quando comparado aos teores relativos às outras três vitaminas (maior capacidade do processo). Tendo em vista o valor alcançado de $C_{p K}$ para o teor de pantotenato de cálcio, o processo de mistura pode ser considerado validado. No que se refere à probabilidade de falhas, valores inferiores ao LIE e superiores ao LSE foram determinados como sendo menor que uma ocorrência em 1 milhão de lotes fabricados.

Na Tabela II estão apresentados os índices de capacidade dos processos relativos ao teor de vitaminas na mistura. Os valores de $C_{p k}$ indicaram que o processo apresentou maior capacidade para o teor de pantotenato de cálcio $\left(C_{p k}\right.$ igual a 1,96$)$. Esse resultado pode ser fundamentado no maior tempo de mistura para essa vitamina (38 minutos), conforme Figura 1. No caso do cloridrato de piridoxina, com o mesmo tempo de mistura, a capacidade do processo foi a menor quando comparada às outras três vitaminas $\left(C_{p k}\right.$ igual a 1,01$)$. Tal resultado pode ser decorrente da concentração desse componente na mistura $(10,456 \mathrm{~g} / \mathrm{kg})$, a menor entre as três vitaminas (Tabela I). Dessa forma, estudos adicionais devem ser conduzidos visando à otimização do tempo de mistura para esse componente. No caso do ácido ascórbico $\left(C_{p k}\right.$ igual a 1,21$)$ e da nicotinamida $\left(C_{p k}\right.$ igual a 1,14$)$, o tempo de mistura igual 23 minutos e a maior proporção dessas vitaminas na mistura: $82,115 \mathrm{~g} / \mathrm{kg}$ e $492,691 \mathrm{~g} / \mathrm{kg}$, respectivamente, para a nicotinamida e para o ácido ascórbico, constituem importantes fatores para a adequada homogeneidade dos lotes produzidos.

Com referência à não-centralização dos valores obtidos, tal comportamento deve ser investigado empregando ferramentas de qualidade específicas. $\mathrm{O}$ esforço nesse sentido permitiria a obtenção de índices $C_{p}$ e $C_{p k}$ maiores e, como conseqüência, redução na probabilidade de falhas no processo, para todas as vitaminas avaliadas.

\section{CONCLUSÃO}

Os resultados permitiram concluir que o processo de fabricação do produto constituído por mistura polivitamínica pode ser considerado validado segundo abordagem retrospectiva. Os índices de capacidade $\left(C_{p k}\right)$ para as vitaminas avaliadas foram superiores a $1 \mathrm{e}$ iguais a 1,01,1,14, 1,21 e 1,96, respectivamente, para o cloridrato de piridoxina, nicotinamida, ácido ascórbico e pantotenato de cálcio. Além disso, investigações devem ser conduzidas visando à centralização da concentração teórica do teor de vitaminas, na faixa relativa aos limites de controle do processo. Nesse caso, sua probabilidade de falha poderia ser reduzida (maior $C_{p k}$ ). No caso do cloridrato de piridoxina $\left(C_{p k}\right.$ igual a 1,01), além de estudos relativos à centralização da concentração teórica, o tempo de mistura poderia ser otimizado visando maior homogeneidade da mistura.

\section{ABSTRACT}

\section{Statistical approach on retrospective validation process of polyvitaminic mixture}

The regulatory demands related to the labeling of food, mainly the one constituted of polyvitaminics and minerals destined to lactants and children in their first infancy, require manufactured process that assure the labeled content. Therefore, the suppliers of polyvitaminics have to demonstrate that the process of powder homogeneity leads to expected results, according to the specifications. Thus, the objective of this work was to validate the process of the mixture of the following vitamins: pyridoxine hydrochloride, nicotinamide, ascorbic acid and calcium pantothenate, based on retrospective approach. Control charts were employed for the statistic analysis of the data related to the assay of each vitamin targeting the evaluation of the stability of the process. The capacity indexes, $C_{p}$ and $C_{p k}$, were calculated after the evaluation 
of the data distribution using Anderson-Darling test. All data showed normal distribution, except for nicotinamide assay. This data was treated by a method proposed by BoxCox (lambda equal -4.24) aimed at obtaining the normality of the data. The capacity indexes $\left(C_{p k}\right)$ obtained were 1.01, 1.14, 1.21 and 1.96, respectively, for pyridoxine hydrochloride, nicotinamide, ascorbic acid and calcium pantothenate. Therefore, the process of polyvitaminic mixture can be considered validated.

UNITERMS: Polyvitaminic. Validation. Stability. Normality.Capacity.

\section{REFERÊNCIAS BIBLIOGRÁFICAS}

BOLTON, S.; BON, C. Pharmaceutical statistics: practical and clinical applications. New York: Marcel Dekker, 2004. 755p. (Drugs and the pharmaceutical sciences, v.135).

BRASIL. Ministério da Saúde. Agência Nacional de Vigilância Sanitária. Legislação. Visalegis. Resolução RDC n.210, de 04 de agosto de 2003a. Determina a todos os estabelecimentos fabricantes de medicamentos, o cumprimento das diretrizes estabelecidas no Regulamento Técnico das Boas Práticas para a Fabricação de Medicamentos, conforme ao Anexo I da presente Resolução. Disponível em: <http://e-legis.bvs.br/leisref/ public/showAct.php?id=22321\&word=>. Acesso em: 08 abr. 2005.

DUDEK-BURLIKOWSKA, M. Quality estimation of process with usage control charts type $\mathrm{X}-\mathrm{R}$ and quality capability of process $\mathrm{C}_{p}, \mathrm{C}_{p k}$. J. Mat. Process. Technol., v.162-163, p.736-743, 2005.
MONTGOMERY, D.C. Introdução ao controle estatístico da qualidade. 4.ed. Rio de Janeiro: LTC, 2004. 513p.

PEARN, W.; LIN, P. Testing process performance based on capability index $\mathrm{C}_{p k}$ with critical values. Comput. Ind. Eng., v.47, p.351-369, 2004.

RAMOS, A.W. Uma contribuição aos estudos de capacidade de máquina. São Paulo, 1999.298p. [Tese de Doutorado. Escola Politécnica. Universidade de São Paulo].

SHEWHART, W.A. Economic control of quality of manufactured product. New York: Van Nostrand, 1931. $501 \mathrm{p}$.

SRIKAEO, K.; FURST, J.; ASHTON, J. Characterization of wheat-based biscuit cooking process by statistical process control techniques. Food Control, v.16, p.309-317, 2005.

STOUMBOS, Z.G. Process capability indices: overview and extensions. Nonlinear Anal., v.3, n.2, p.191-210, 2002.

UNITED STATES Pharmacopeia: USP28; National Formulary: NF23. Rockville: United States Pharmacopeial Convention, 2005. p.178, 331,13691370,1683-1684.

UNITED STATES. Food and Drug Administration. Guidelines on general principles of process validation of 1987. 12p. Disponível em: <http://www.fda.gov/cdrh/ode/ 425.pdf>. Acesso em: 30 mar. 2005.

Recebido para publicação em 10 de março de 2006. Aceito para publicação em 03 de janeiro de 2007. 